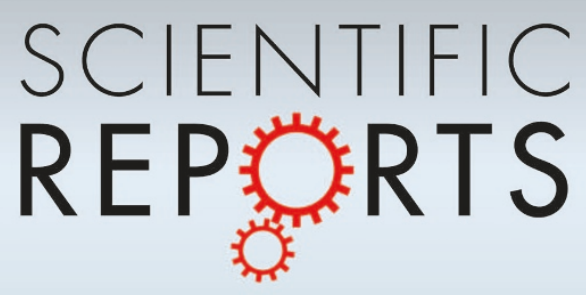

OPEN

SUBJECT AREAS:

BIOSENSORS

NANOSENSORS

BIOANALYTICAL CHEMISTRY

Received

21 August 2013

Accepted

12 November 2013

Published

28 November 2013

Correspondence and requests for materials should be addressed to H.A.C. (h.clark@neu.

edu)

\footnotetext{
* These authors contributed equally to this work.
}

\section{Polymer-Free Optode Nanosensors for Dynamic, Reversible, and Ratiometric Sodium Imaging in the Physiological} Range

\author{
Timothy T. Ruckh ${ }^{*}$, Ankeeta A. Mehta' ${ }^{1 *}$, J. Matthew Dubach² \& Heather A. Clark'
}

\begin{abstract}
'Department of Pharmaceutical Sciences, Northeastern University, 360 Huntington Avenue, Boston, MA $02115,{ }^{2}$ Department of Bioengineering, Northeastern University, 360 Huntington Avenue, Boston, MA 02115.
\end{abstract}

This work introduces a polymer-free optode nanosensor for ratiometric sodium imaging. Transmembrane ion dynamics are often captured by electrophysiology and calcium imaging, but sodium dyes suffer from short excitation wavelengths and poor selectivity. Optodes, optical sensors composed of a polymer matrix with embedded sensing chemistry, have been translated into nanosensors that selectively image ion concentrations. Polymer-free nanosensors were fabricated by emulsification and were stable by diameter and sensitivity for at least one week. Ratiometric fluorescent measurements demonstrated that the nanosensors are selective for sodium over potassium by $\sim 1.4$ orders of magnitude, have a dynamic range centered at $20 \mathrm{mM}$, and are fully reversible. The ratiometric signal changes by $70 \%$ between 10 and $100 \mathrm{mM}$ sodium, showing that they are sensitive to changes in sodium concentration. These nanosensors will provide a new tool for sensitive and quantitative ion imaging.

on channels, though largely associated with the study of excitable cells such as neurons ${ }^{1}$ and myocytes ${ }^{2}$, play key roles in a broad spectrum of physiological processes including cancer cell proliferation ${ }^{3,4}$ and inflammation $^{5}$. Whole-cell and single-channel electrophysiology recordings are standard techniques to characterize channel biophysical properties and a suite of calcium indicator dyes (e.g. Fluo, Fura, Indo) can report spatiotemporal calcium dynamics with fluorescence microscopy ${ }^{6}$. However, indicator dyes for sodium (SBFI, CoroNa, Asante Natrium Green) and potassium (PBFI) are less widely used, due in part to their selectivity, photostability, and short wavelengths. A new, robust fluorescent sodium or potassium sensor could elucidate the connection between disease-linked ion channel mutations or abnormal expression patterns and intracellular signaling.

Ion-selective optodes, the optical equivalent of ion-selective electrodes ${ }^{7,8}$, provide an attractive platform for designing new intracellular nanosensors. Their general formulation contains a matrix of plasticized polymer, a pH-responsive chromoionophore, an optically inactive ionophore, and an optically-inactive hydrophobic charge-carrying molecule to facilitate ion exchange within the sensor's hydrophobic core ${ }^{9-11}$. The ionophore selectively carries an ion, sodium for example, into the core, and that sodium ion deprotonates the chromoionophore in order to maintain charge neutrality in the core. The chromoionophore's absorbance and fluorescence reflect this protonation change, thus connecting the sodium concentration to the sensor's optical properties.

Previous works from our group directly translated bulk optodes into nanosensors for real-time, single-cell, bioanalytical chemistry ${ }^{12-16}$. In those works, the matrix supporting the sensing components contained $\sim 33 \%$ polymer and $\sim 66 \%$ plasticizer. The polymer imparts mechanical stability while the plasticizer allows the three encapsulated sensing components to diffuse within an individual nanoparticle. Although the polymer's mechanical stability is advantageous during fabrication, removing the polymer from the formulation may eventually allow further size reductions. This could be key for using nanosensors for intracellular experiments, where small compartments such as dendritic spine necks may be as narrow as $100 \mathrm{~nm}$ in diameter ${ }^{17}$.

For intracellular scenarios, a nanosensor must sense the ion of interest in a complex environment. Here, sodium is the ion of interest and potassium is the primary interfering ion. Intracellular potassium levels are $\sim 140 \mathrm{mM}$ for mammalian cells, which is sufficient to interfere with common fluorescent sodium indicators such as CoroNa ${ }^{18}$. Lamy et al. (2011) improved CoroNa's intracellular retention and stability by encapsulating the molecule within a fifth-generation poly(amido amine) (PAMAM) dendrimer and imaging dendritic sodium 
accumulation in brain slices ${ }^{19}$. Other researchers have had varying degrees of success with SFBI or Sodium Green ${ }^{20}$, but all three sodium indicators lack sensitivity and have low signal-to-noise. Furthermore, only SFBI is capable of ratiometric measurements, and it requires either single-photon excitation with expensive UV lasers ${ }^{21}$ or two-photon optics ${ }^{22}$. To improve signal strength and provide ratiometric measurement we designed new, polymer-free nanosensors (PFNs) containing two dynamic fluorophores that emit with peaks at 580 and $680 \mathrm{~nm}$.

\section{Results}

In this work we demonstrate that polymer-free optode nanosensor (PFN) formulations are functional, stable, and tuned to be sodiumselective in a physiologically-relevant environment. In traditional optode-based sensors, the supporting matrix contains $\sim 33 \%$ polymer for stability and $\sim 66 \%$ plasticizer to allow the sensing components to freely diffuse within the optode and extract the analyte of interest. This stability is also beneficial when translating optode sensors into nanosensors through an emulsification step. As long as the emulsification step is relatively brief ( $\sim 1 \mathrm{~min})$, PFNs with protonated chromoionophore are attainable and they appear blue.

We first demonstrated stability by two measures; response stability to show that PFNs maintain their sensitivity and size stability to show that PFNs do not aggregate. Calibrations for sodium responses through 8 days showed that the ratiometric measurement had a very stable response (Figure 1A). On day 0, PFNs detected sodium with a dissociation constant, $\mathrm{K}_{\mathrm{d}}$, of $5.9 \pm 0.5 \mathrm{mM}$ (Figure $\mathrm{S} 1$ ), and that $\mathrm{K}_{\mathrm{d}}$ changed to $3.7 \pm 0.2 \mathrm{mM}$ by day 8 (Figure $1 \mathrm{~B}$ ). The largest change in $\mathrm{K}_{\mathrm{d}}$ occurred between the first two days of the study, and although the statistical model determined that the effect of time was statistically significant $(\mathrm{p}<0.0001)$, its effect only reduced the $\mathrm{K}_{\mathrm{d}}$ by $2.2 \mathrm{mM}$ overall. Thus, PFN sensitivity is very reliable through 8 days if stored at room temperature and shielded from light.

Both fluorophores, octadecyl rhodamine (Rhd18) and chromoionophore III (CHIII) also retained their sensitivity during the eightday experiment. The fluorescent response for Rhd18 and CHIII over $1 \mathrm{log}$ unit of sodium, between $10 \mathrm{mM}$ and $100 \mathrm{mM}$, changed very minimally (Figure S1A), and the statistical effect was insignificant (Rhd18: $\mathrm{p}=0.72$; CHIII: $\mathrm{p}=0.21$ ). Interestingly, the ratio of CHIII : Rhd18 did decrease at a statistically significant level $(\mathrm{p}<$ 0.0001 ), but the change in sensitivity was only $6 \%$ over the 8 days (Figure S1B). These results indicate that if the local sodium concentration were to change from $10 \mathrm{mM}$ to $100 \mathrm{mM}$, the ratiometric measurement of CHIII fluorescence to Rhd18 fluorescence would be approximately $6 \%$ less on day 8 than on day 0 .

The sensitivity is defined as the slope of the log-linear sensor range. Between 10-100 mM sodium Rhd18 increased with a slope $8000 \mathrm{RFU} / \log \left[\left(\mathrm{Na}^{+}\right)\right]$, and its fluorescence increased by $103 \%$. Over the same range, CHIII decreased with a slope of $3900 \mathrm{RFU} /$ $\log \left(\left[\mathrm{Na}^{+}\right]\right)$, and its fluorescence decreased by $40 \%$. The ratio of the CHIII : Rhd18 had a slope of $0.08 / \log \left[\left(\mathrm{Na}^{+}\right)\right]$, and it decreased by $71 \%$. Altogether, PFNs exhibit excellent sensitivity to changes is sodium concentrations relevant to mammalian cells and retain their sensitivity for up to eight days at room temperature.

For PFNs to function as probes for localized ion fluxes, they must remain nano-scale in size and not spontaneously agglomerate. Size stability was measured by DLS when PFNs were stored at room temperature for eight days. Immediately after emulsification, PFNs showed an effective diameter of $254 \mathrm{~nm}$ (Figure $2 \mathrm{~A}$ ), and by the $8^{\text {th }}$ day the mean diameter had decreased by only $8 \mathrm{~nm}(\mathrm{p}=0.001)$. Although this effect was statistically significant, the actual change was very small and the results demonstrate that PFNs are stable nano-emulsions for up to eight days.

The smallest PFN size population was isolated by a centrifugation step. Four different centrifugation speeds $-2500,5000,7500$, or $10,000 \times \mathrm{g}-$ and four time points $-3,6,9$, or 12 minutes - each provided a different but predictable trend for isolating PFNs of a particular size. Prior to centrifugation, all samples measured $264 \mathrm{~nm}$ (Figure 2B), and, predictably, faster centrifugation speeds and longer times reduced the effective diameter. The case of 10,000 $\times$ $\mathrm{g}$ for 12 minutes provided the only large deviation from this trend, as that case actually increased the measured particle size from $130 \mathrm{~nm}$ to $150 \mathrm{~nm}$. Based on these results, PFNs with effective diameters anywhere from $130-260 \mathrm{~nm}$ are easily attainable.

The $\mathrm{K}_{\mathrm{d}}$ for the smaller PFNs was then compared to un-centrifuged PFNs by centrifuging PFNs for 9 minutes at 2500, 5000, 7500, or $10,000 \times \mathrm{g}$ and then measuring their responses to sodium. The uncentrifuged PFNs had a $\mathrm{K}_{\mathrm{d}}$ of $4.5 \mathrm{mM}$, which was significantly different $(\mathrm{p}<0.0001)$ from all other treatments (Figure $2 \mathrm{C}$ ). All of the measured $K_{d}$ values for centrifuged PFNs were between 15-25 mM, though there was a significant difference between the 2500 and 7500 $\times$ g preparations.

In order to characterize PFN selectivity for sodium over potassium, the primary intracellular interfering ion, $\mathrm{PFN}$ responses to sodium were measured in the presence of a range of static potassium concentrations (Figure $3 \mathrm{~A}$ ). The $\mathrm{K}_{\mathrm{d}}$ did not change between 1$50 \mathrm{mM}$ potassium, but the increase in $\mathrm{Kd}$ due to 150 and $333 \mathrm{mM}$ potassium was significant $(\mathrm{p}<0.0001)$ (Figure $3 \mathrm{~B})$. The low and high ends of physiologically-relevant ranges for most mammalian cells spans $4 \mathrm{mM}$ and $150 \mathrm{mM}$ for sodium and potassium. To calculate the selectivity coefficient for sodium over potassium, PFN responses to potassium in the presence of $4 \mathrm{mM}$ and $150 \mathrm{mM}$ background sodium were measured and fit to the Nicolskii-Eisenman model (Equation 3). In $4 \mathrm{mM}$ and $150 \mathrm{mM}$ background sodium, PFNs responded to potassium with a $\mathrm{K}_{\mathrm{d}}$ of $543 \mathrm{mM}$ and $1785 \mathrm{mM}$, respectively. This leads to a selectivity coefficient,

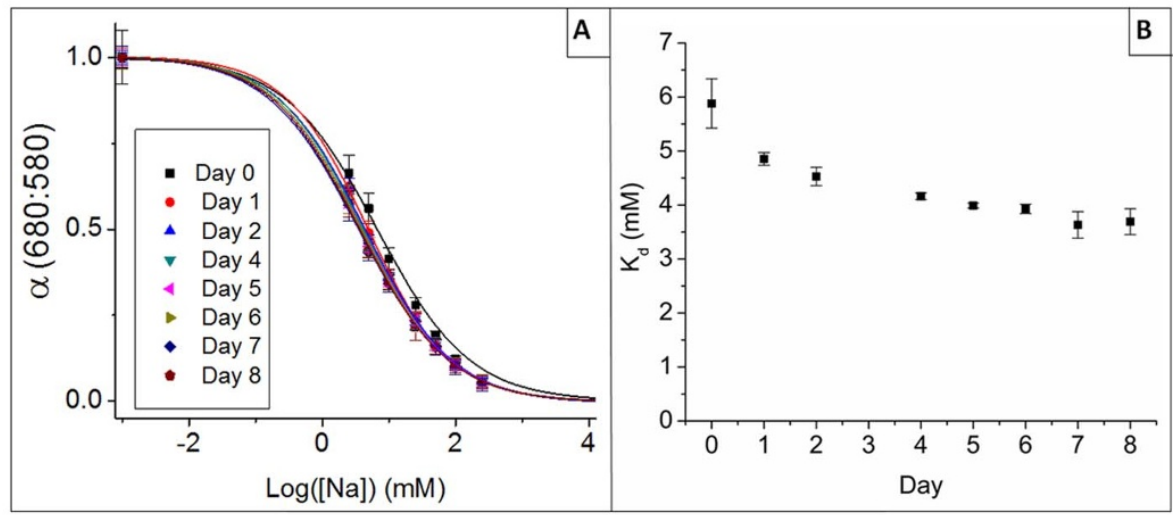

Figure 1 Calibration curves (A) for PFNs showing small changes in sensitivity to sodium through eight days in storage at room temperature. The calculated $K_{d}$ values (B) for PFNs at each of the eight days. Data represented as mean values with error bars for standard deviations. 


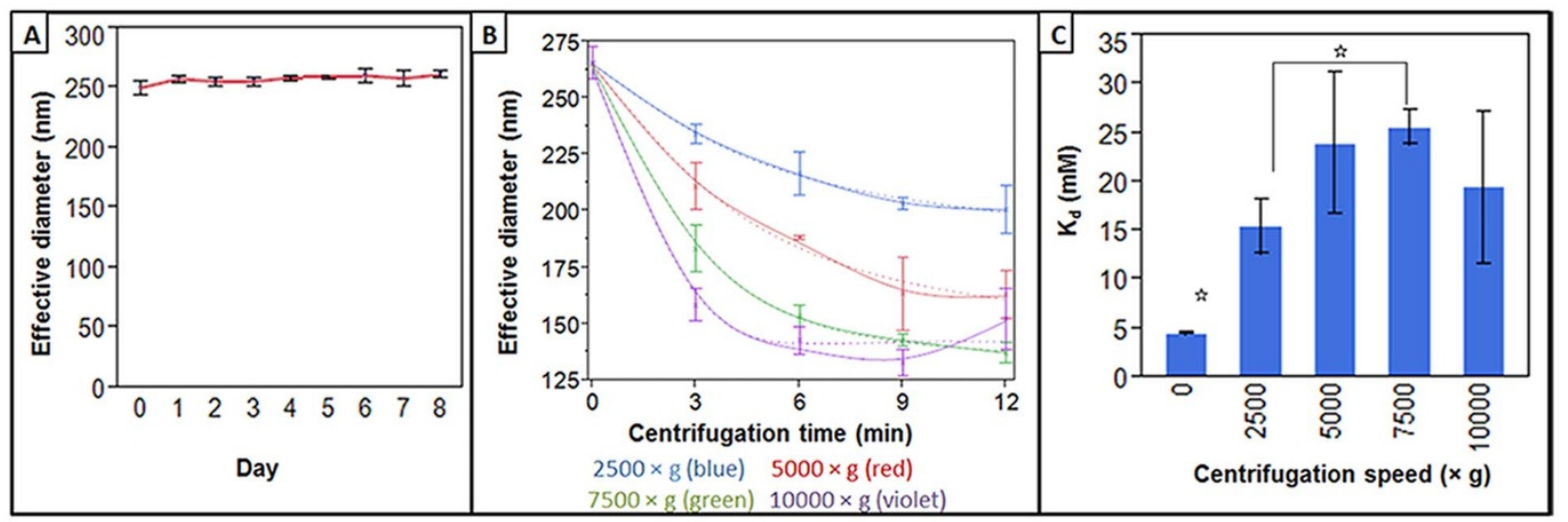

Figure 2 Size stability of PFN (A) stored for eight days at room temperature, measured by DLS. Freshly-made PFNs can be separated for size by centrifugation (B) in a manner dependent on centrifugation speed and time. After centrifugation for 9 minutes, PFNs were calibrated to characterize any effect on their sensitivity (C). Data represented as mean values with error bars for standard deviation.

reported as $\log \left(K_{\mathrm{Na}, K}^{\text {opt }}\right)$, of -1.09 in $4 \mathrm{mM}$ interfering ion (Figure $\mathrm{S} 3 \mathrm{~A}$ ) and -1.43 in $150 \mathrm{mM}$ interfering ion (Figure S3B). For intracellular analysis, the $150 \mathrm{mM}$ case is the most relevant, and the $\mathrm{K}_{\mathrm{d}}$ for potassium in both cases means that the effects of physiologicallyrelevant potassium dynamics have been minimized.

PFN responses when both fluorophores are simultaneously excited were measured on a confocal microscope by first loaded dialysis tubing with PFNs and then imaging the PFNs in 0$1000 \mathrm{mM}$ sodium (Video S1). Both fluorophores responded dynamically to changes in sodium concentration (Figure $4 \mathrm{~A}$ ), and the ratio of the two fluorophores, measured as $680: 580$, changed by $87 \%$ between $0 \mathrm{mM} \mathrm{NaCl}$ and $100 \mathrm{mM} \mathrm{NaCl}$ (Figure 4B). PFNs were also responsive to physiologically-relevant changes as well; the fluorescence ratio changed by nearly $60 \%$ between 8 and $100 \mathrm{mM}$ sodium. The calibration data also fit the Hill equation (Figure S4) and produced a $K_{d}$ of $10.9 \mathrm{mM}$ for the ratio of $680: 580 \mathrm{~nm}$. This $K_{d}$ value is lower than the values measured in calibrations performed on a plate reader, but this is likely a consequence of exciting both fluorophores simultaneously since CHIII has some absorption from $555 \mathrm{~nm}$ laser excitation and the 580 Rhd18 emission.

Reversibility is key to characterizing sodium influx parameters similar to ones made for calcium sparks, such as the rise and decay times $^{23}$. To demonstrate reversibility, and to simulate reversible sodium fluctuations ${ }^{14,24-26}$, short sections of dialysis tubing containing unknown amounts of PFNs were mounted on a microscope flow chamber and images were recorded at 10 second intervals (Video S2). The medium cycled between solutions of 10 or $100 \mathrm{mM} \mathrm{NaCl}$ in
$10 \mathrm{mM}$ HEPES. The profile of mean fluorescence for each fluorophore (Figure 5A) illustrates the variability due to differences in PFN concentration between batches, but reversibility is still evident. The ratio of fluorescence from the two fluorophores, CHIII: Rhd18 shows the benefits of a ratiometric nanosensor (Figure 5B). Most importantly, though, the sensors reverse and can go through many cycles with repeatable performance.

\section{Discussion}

The shortcomings of existing indicator dyes for imaging sodium or potassium have been discussed elsewhere ${ }^{22}$, and new ion imaging reagents are a known need. The new sensor presented here is simple to fabricate and nanosensors with hydrodynamic radii $\sim 130 \mathrm{~nm}$ are easy to isolate from the crude product (Figure 2B). There was a noted increase in $\mathrm{K}_{\mathrm{d}}$ from $4 \mathrm{mM}$ to $20 \mathrm{mM}$ when smaller PFNs were separated by centrifugation (Figure 2C). For intracellular analysis, a $\mathrm{K}_{\mathrm{d}}$ of $20 \mathrm{mM}$ is preferable over a $\mathrm{K}_{\mathrm{d}}$ of $4 \mathrm{mM}$ because the resting intracellular sodium concentration is $5-12 \mathrm{mM}$ in most mammalian cells $^{14,27-29}$. If higher $K_{d}$ values were of interest, for example in a squid giant axon, then the PFN formulation should contain more chromoionophore relative to ionophore so that the PFNs are still responsive to sodium influxes of up to $400 \mathrm{mM}^{30}$. This tunability is a key advantage over molecular dyes that would require chemical modification to change their sensitivity.

Nanosensors must respond linearly to sodium with high sensitivity and selectivity over potassium and other ions. $A K_{d}$ value within the expected range of values will produce a linear sensor response.

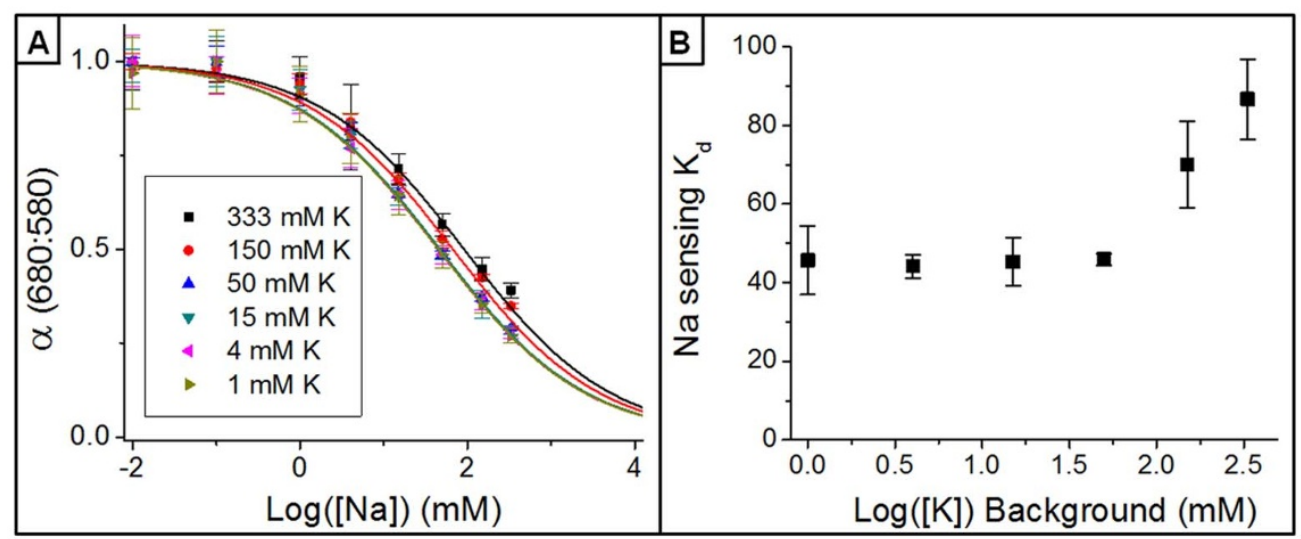

Figure 3 PFN calibration performed in 1-333 mM potassium background (A). $K_{d}$ values calculated for sodium response in 1-333 mM potassium background (B). Data represented as mean with error bars for standard deviation. 


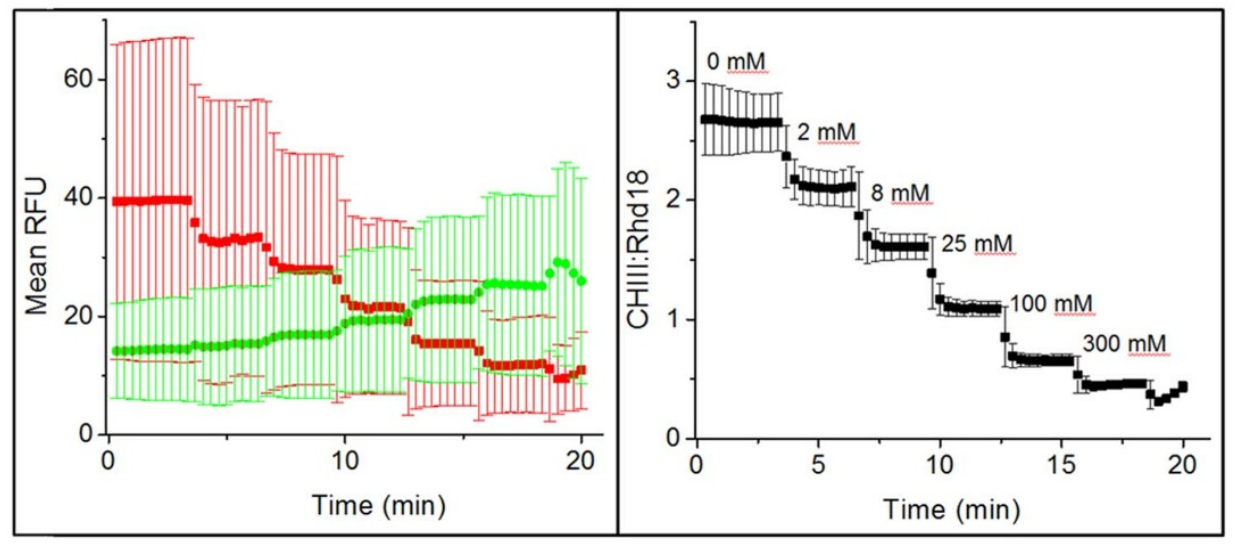

Figure 4 Calibration experiment performed with PFNs loaded into a micro-dialysis tube at unknown concentrations and imaged on a confocal microscope. Solutions of 0-1000 mM NaCl were washed through, and the mean fluorescent intensity for Rhd18 (green, $580 \mathrm{~nm}$ ) and CHIII (red, $680 \mathrm{~nm}$ ) were measured simultaneously (A). The ratio for CHIII : Rhd18 (680:580) was computed in each frame and presented (B) for the same image set.

After separating sub-200 $\mathrm{nm}$ nanoparticles, PFN $\mathrm{K}_{\mathrm{d}}$ values were approximately $20 \mathrm{mM}$, which is nearly ideal for intracellular analysis. One recent study determined that volumetric sodium concentrations only change by a few $\mathrm{mM}$ from baseline during action potentials ${ }^{31}$, and these sensors are well-suited to measure changes is sodium concentrations around 15-20 mM (Figure 3).
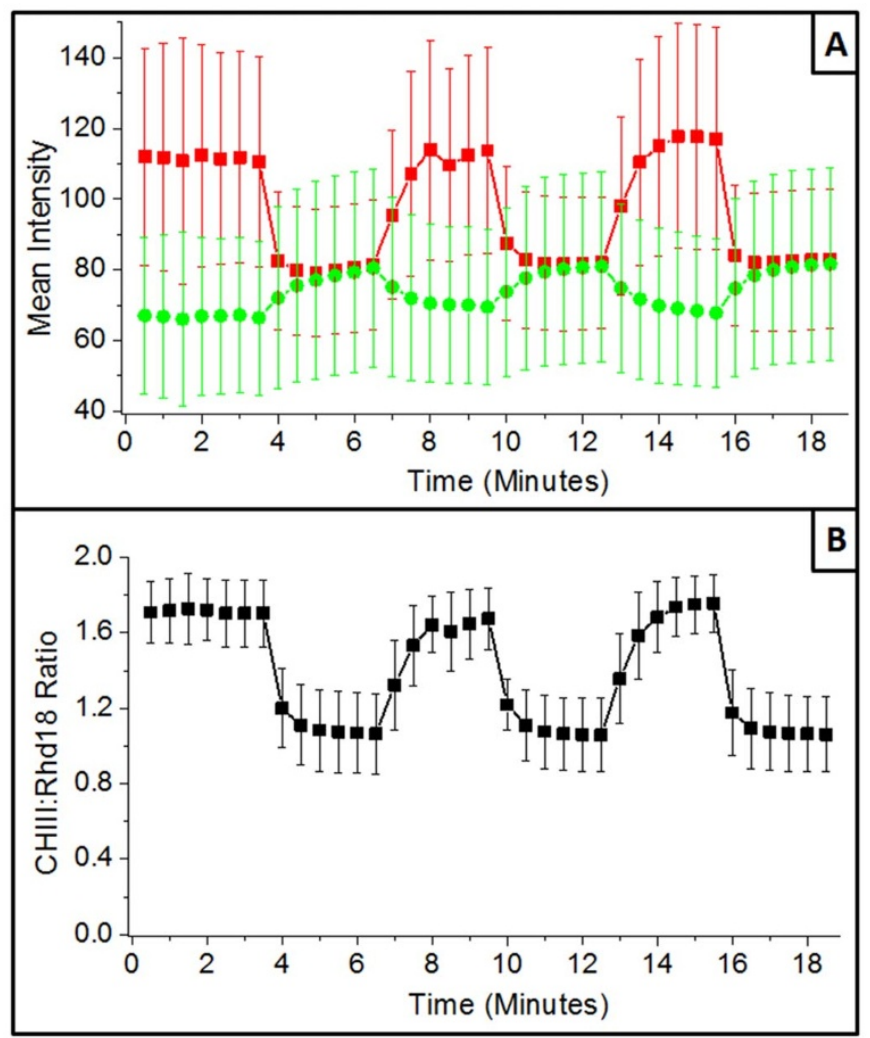

Figure 5 Reversibility experiment performed with PFNs loaded into a micro-dialysis tube. Solutions of 10 or $100 \mathrm{mM} \mathrm{NaCl}$ were washed through and the mean fluorescent intensity imaged in the dialysis tubing for Rhd18 (green, $580 \mathrm{~nm}$ ) and CHIII (red, $680 \mathrm{~nm}$ ) were measured separately (A). The ratio for CHIII : Rhd18 $(680: 580)$ was computed in each frame and presented (B) for the same image set, showing the reversibility through three cycles and 19 minutes of imaging.
PFNs also compare favorably to other sodium indicators like CoroNa Green and SBFI. One recent approach to encapsulate CoroNa Green within a sixth generation poly(amido amine) dendrimer determined that the system had a $\mathrm{K}_{\mathrm{d}}$ of $81.2 \mathrm{mM}$, which is very close to another independent calibration of the molecular dye itself. $\mathrm{SBFI}^{19,32}$, a ratiometric sodium indicator, has a lower $\mathrm{K}_{\mathrm{d}}$ of 10 $20 \mathrm{mM}$, but its emission peaks are both below $400 \mathrm{~nm}^{32,33}$ and that limits its effective depth within a tissue. SBFI is also limited by its low quantum yields of 0.08 and 0.045 in its sodium-bound and free forms, respectively ${ }^{34}$, while Rhd18 and CHIII have quantum yields of nearly $0.6^{35}$ and $0.25^{36}$, respectively. Additionally, PFNs respond to sodium in both intracellular and extracellular concentrations, while SBFI is known to saturate around $100 \mathrm{mM}$ sodium and is not suitable for measuring extracellular sodium ${ }^{37}$. Due to these sufficient drawbacks to existing sodium indicators, PFNs are a significant improvement for quantitatively measuring physiologically-relevant sodium concentrations.

Selectivity must always be demonstrated with optode-based ion sensors. The ionophore used here, sodium ionophore $\mathrm{X}$, has been robustly characterized for use in ion selective electrodes ${ }^{38,39}$ and in ion-selective optodes ${ }^{40,41}$. Based on those reports, the primary interfering ion for this sensor is potassium, and thus we determined the selectivity coefficient for this sensor formulation to be 1.09-1.43 depending on the background ionic strength (Figure S3A and $\mathrm{S} 3 \mathrm{~B})$. This ionophore is at least one additional log unit more selective over calcium than it is over potassium ${ }^{38-41}$, so although calcium concentrations could change dramatically, PFNs will not respond to those changes.

The measured selectivity coefficients are lower than microparticlesized sodium optode sensors that measured $\log \left(K_{\mathrm{Na}, \mathrm{K}}^{\mathrm{opt}}\right)$ values of 2.42.8 using a very similar formulation ${ }^{41}$. Intracellular potassium in excitable cells drops only by $20-25 \mathrm{mM}$, even during long-term or repeated depolarizations such as cortical spreading depression models ${ }^{42}$, ischemia $^{43}$, or high-intensity exercise ${ }^{44}$. Furthermore, the actual change in 680:580 emission in response to potassium changes $4-150 \mathrm{mM}$ is quite small. If sodium remained constant as potassium levels changed from 140 to $115 \mathrm{mM}$, the observable change in $680: 580$ would be only $\sim 7 \%$ of the signal and indicate a 'false' sodium increase of $9 \mathrm{mM}$. Thus for the conditions of intracellular analysis, these PFNs are much more sensitive to sodium changes than potassium changes.

Perhaps the most attractive feature of the PFN design is the ratiometric signal, which is important for scenarios when sensor loading is difficult to control. The measurement of $680: 580$ proved to be independent of nanosensor concentration (Figures $4 \mathrm{~B}$ and 5B). Additionally, CHIII and Rhd18 are both environmentally-sensitive 
to $\mathrm{pH}$, but in opposite directions. At an acidic $\mathrm{pH}$ in hydrophobic media, Rhd18 fluorescence is low, but in more basic conditions its fluorescence increases dramatically ${ }^{45}$. Conversely, CHIII is highly protonated in acidic conditions and fluoresces strongly while it does not fluoresce well when it is deprotonated ${ }^{9,11}$. Having two dynamic fluorophores (Figures $4 \mathrm{~A}$ and $5 \mathrm{~A}$ ), rather than one as a static reference fluorophore, increased the ratiometric measurement sensitivity (Figures $4 \mathrm{~B}$ and $5 \mathrm{~B}$ ).

In this work, we designed and validated optical nanosensors that are selective for sodium. By implementing a design with nanoparticles composed of polymer-free optode components and two dynamic fluorophores, we produced sensors with a ratiometric signal and the ability to selectively and reversibly measure sodium. The sensors are bright, shelf-stable, and size-separable with a standard benchtop centrifuge. Simply using an appropriate ratio of sensing components and separation, PFNs with a $\mathrm{K}_{\mathrm{d}}$ of $15-25 \mathrm{mM}$ and reversible sodium responsiveness were produced. Future work will focus on applying these sensors to electrophysiological studies of excitable cells.

\section{Methods}

Materials. Bis(2-ethylhexyl) sebacate (DOS), chromoionophore III (CHIII), sodium ionophore $\mathrm{X}$ (NaIX, 4-tert-butylcalix[4] arene-tetraacetic acid tetraethyl ester), Sodium tetrakis[3,5-bis(trifluoromethyl)phenyl]borate (NaTFPB), 4-(2Hydroxyethyl)piperazine-1-ethanesulfonic acid (HEPES), sodium chloride $(\mathrm{NaCl})$, 90,000 MW poly(vinyl chloride) (PVC), dichloromethane (DCM) and tetrahydrofuran (THF) were purchased from Sigma-Aldrich. Octadecyl rhodamine B chloride (Rhd18) was purchased from Life Technologies. Tris Base was purchased from Fisher BioReagents. Spectra/Por in vivo micro-dialysis hollow fibers (inner diameter $200 \mu \mathrm{m}$; outer diameter $280 \mu \mathrm{m}$, MWCO $13 \mathrm{kD}$ ) were purchased from Spectrum Labs, Inc. 1,2-distearoyl-sn-glycero-3-phosphoethanolamine-N[methoxy(polyethylene glycol)-550] (ammonium salt) (DSPE-mPEG550) was purchased from Avanti Polar Lipids, Inc.

Polymer-free nanosensor (PFN) fabrication. First, the sensing components NaIX $(2 \mu \mathrm{mol}, 2 \mathrm{mg})$, CHIII ( $878 \mathrm{nmol}, 0.5 \mathrm{mg})$, NaTFPB $(1.12 \mu \mathrm{mol}, 1 \mathrm{mg})$, and Rhd 18 (123 nmol, $0.09 \mathrm{mg}$ ) were reconstituted separately with THF, combined in a $2 \mathrm{~mL}$ glass vial, and dried under nitrogen. Just as the THF dried, DOS $(428 \mu \mathrm{mol}, 200 \mu \mathrm{L})$ was added to the sensing components and vortexed for 30 seconds. If the sensing components were completely dried, the resulting PFNs lost fluorescence and responsiveness. Just prior to nanosensor fabrication, DSPE-mPEG550 $(500 \mu \mathrm{g} / \mathrm{mL}$, $0.05 \% \mathrm{w} / \mathrm{v})$ was dissolved in the HEPES buffer $(2.38 \mathrm{mg} / \mathrm{mL}, 10 \mathrm{mM}$ and the $\mathrm{pH}$ adjusted to 7.2 with tris base) prior to nanosensor fabrication. In a typical PFN fabrication, $40 \mu \mathrm{L}$ of polymer-free optode was added to $4 \mathrm{~mL}$ of HEPES buffer containing $0.05 \%$ DSPE-mPEG550 in a glass vial. The mixture was sonicated for 1 minute with a Branson digital sonifier (S-450D) at $10 \%$ intensity with a $1 / 8$ " diameter tip. If PFNs are sonicated for longer than 1 minute at $10 \%$ amplitude, the chromoionophore becomes deprotonated.

Plasticized polyvinyl chloride (PVC) nanosensor fabrication. Nanosensors with a matrix of plasticized PVC were made as previously reported ${ }^{12,14-16}$. Briefly, PVC (36 nmol, $3.3 \mathrm{mg})$, DOS $(141 \mu \mathrm{mol}, 66 \mu \mathrm{L})$, NaIX $(200 \mu \mathrm{mol}, 300 \mu \mathrm{g})$, CHIII $(88 \mathrm{nmol}, 50 \mu \mathrm{g})$, Rhd18 (12 nmol, $9 \mu \mathrm{g})$, and NaTFPB (112 nmol, $100 \mu \mathrm{g})$ were dissolved in $50 \mu \mathrm{L}$ of THF in the same manner as PFNs. $70 \mu \mathrm{L}$ DCM was added to the mixture, and it was sonicated for 3 minutes at $15 \%$ intensity in $4 \mathrm{~mL}$ of pH $7.2 \mathrm{HEPES}$ buffer $(2.38 \mathrm{mg} / \mathrm{mL}, 10 \mathrm{mM})$ containing DSPE-mPEG550 $(500 \mu \mathrm{g} / \mathrm{mL}, 0.05 \% \mathrm{w} / \mathrm{v})$. The resulting solution was filtered with a $0.2 \mu \mathrm{m}$ syringe filter (Millipore).

Particle size measurements. Hydrodynamic diameters were measured by dynamic light scattering (DLS) using a 90 Plus particle size analyzer by Brookhaven Instruments Corporation. All particle size measurements were taken in triplicate by diluting the sample solution in 10 mM HEPES buffer, pH-7.2 so that the detector count rate measured 50-450 kcps. The detector measured scattered $640 \mathrm{~nm}$ light at a fixed angle of $90^{\circ}$, and calculated the effective diameter based on the intensity of scattered light.

PFN Size Separation. Solutions of freshly-prepared PFNs were centrifuged for 0, 3, 6, 9 , and 12 minutes at $2500,5000,7500$, or $10,000 \times g$ at room temperature. At each time point, $40 \mu \mathrm{L}$ or PFN solution was removed and prepared for size measurement by dynamic light scattering, as described below. Data were analyzed to test for the individual effects of time and centrifugation speed and the interaction of centrifugation speed by time.

Size-dependent response to sodium. Freshly-prepared PFNs were centrifuged for 9 minutes at $2500,5000,7500$, or $10,000 \times g$ at room temperature. Each sample was then calibrated for its fluorescent response to sodium in $10 \mathrm{mM}$ HEPES buffer, $\mathrm{pH}$ 7.2. For each calibration point, the PFN solution was mixed with standard solutions so that the final $\mathrm{NaCl}$ concentrations were $0-500 \mathrm{mM} \mathrm{NaCl}$ and a total of 8 calibration points. $500 \mathrm{mM} \mathrm{NaOH}$ or $\mathrm{HCl}$ were used to, respectively, fully deprotonate or protonate the chromoionophore. The fluorescence intensities for Rhd18 (ex: 555, em: 580) and CHIII (ex: 639, em: 680) were measured with Spectramax M3 plate reader (Molecular Devices) in bottom read mode through clear-bottom 96-well plates. The fluorescence ratio for CHIII : Rhd18 was calculated as:

$$
R=\frac{680 \text { emission }}{580 \text { emission }}
$$

The ratios for $680 / 580$ were then normalized by computing alpha, defined as:

$$
\alpha=\frac{R_{i}-R_{P}}{R_{D}-R_{P}}
$$

where $\mathrm{R}_{\mathrm{i}}$ is the ratio at a particular $\mathrm{NaCl}$ concentration, $i, \mathrm{R}_{\mathrm{P}}$ is the ratio when the chromoionophore is fully protonated, and $\mathrm{R}_{\mathrm{D}}$ is the ratio when the chromoionophore is fully deprotonated. The dissociation constant, $\mathrm{K}_{\mathrm{d}}$, for the PFNs was calculated from the values of alpha according to the dose-response equation.

PFN selectivity for sodium over potassium. PFN selectivity was determined by measuring the PFN fluorescent response to $0,1,4,15,50,150$, or $333 \mathrm{mM}$ sodium in static concentrations of background potassium. The background potassium concentation for each calibration curve was set to $0,1,4,15,50,150$, or $333 \mathrm{mM}$. Fluorescence intensities for Rhd18 (ex: 555, em: 580) and CHIII (ex: 639, em: 680) were measured on a plate reader and the value of alpha was calculated for the ratio of $680 / 580$ emission intensities as described above. To quantify the PFN selectivity for sodium over potassium, PFNs were also calibrated for their response to potassium in 4 or $150 \mathrm{mM}$ background sodium. The calibration data were fit to the NicolskiiEisenman model with a fixed interfering ion ${ }^{10,46}$, which defines the selectivity coefficient for an optical sensor, $K_{i j}^{o p t}$, as:

$$
\log \left(K_{i j}^{o p t}\right)=\log \left(K_{d}^{j}\right)-\log \left(K_{d}^{i}\right)
$$

$K_{d}^{i}$ and $K_{d}^{i}$ are the $K_{d}$ for the primary and interfering ion, which are sodium and potassium, respectively.

Stability of response and size distribution. In order to characterize the shelf-life for PFNs, their size and fluorescent response to sodium were evaluated over the course of 8 days. In triplicate, PFNs were fabricated and stored at room temperature while protected from exposure to ambient light. At days $0,1,2,4,5,6,7$, and 8 , aliquots were removed to measure the mean particle size, by DLS as described above, and to calibrate the PFN response to sodium. For the calibration, a plate reader read the fluorescent emission for Rhd 18 and CHIII in solutions of $0-500 \mathrm{mM} \mathrm{NaCl}$ in $10 \mathrm{mM}$ HEPES buffer, pH 7.2, as well as $0.5 \mathrm{M} \mathrm{HCl}$ and $\mathrm{NaOH}$ solutions for fully protonated and fully deprotonated controls, respectively. Values for $\mathrm{K}_{\mathrm{d}}$ and particle size were examined using a general linear model to test for the effect of time on either size or $\mathrm{K}_{\mathrm{d}}$.

Calibration and reversibility with confocal microscopy. PFNs were imaged inside of dialysis tubing mounted in a flow chamber for optical microscopy. The setup, described and demonstrated previously ${ }^{46}$, involved filling a $1.5 \mathrm{~cm}$ length of microdialysis tubing (ID: $200 \mu \mathrm{m}$, OD: $250 \mu \mathrm{m}, 13 \mathrm{kDa}$ MWCO, Spectrum Labs) with PFNs suspended in 10 mM HEPES buffer, sealed on its ends with 60-second curing epoxy, and fixed to a $15 \mathrm{~mm}$ diameter circular glass coverslip with two drops of watercuring epoxy. The concentration of PFNs was unknown a priori. The glass coverslip was then secured in a flow chamber (Warner Instruments, RC-21BRFS) and mounted for imaging on a laser scanning confocal microscope (Zeiss LSM 700). For calibration experiments, $555 \mathrm{~nm}$ and $639 \mathrm{~nm}$ laser light excited both fluorophores simultaneously, and the two emissions were filtered with a shortpass filter at $610 \mathrm{~nm}$ (Rhd18) and a longpass filter set to $640 \mathrm{~nm}$ (CHIII). Images were acquired at 30 second intervals and the solutions were changed every 3 minutes. Solutions of $0,2,8$, $25,100,300$ and $1000 \mathrm{mM} \mathrm{NaCl}$ in $10 \mathrm{mM}$ HEPES were used to calibrate the PFNs. Data were then fit to the dose-response equation.

For reversibility experiments, solutions of 10 or $100 \mathrm{mM} \mathrm{NaCl}$ were flowed through the chamber alternatively for 3 exchange cycles. The microscope acquired simultaneous images for Rhd18 and CHIII as the solution changed and images acquired every 30 seconds with the same excitation and emission settings as above. Images were analyzed with the Fiji ${ }^{47}$ plugin suite of Image $(\mathrm{NIH})$ with the region of interest defined as the interior of the dialysis tube and the region of interest definitions for both channels were identical.

Statistical analysis. All experiments were performed in triplicate and analyzed in either Origin 8 (OriginLab) or JMP 10 (SAS). Values are represented numerically as a mean \pm standard deviation, and graphical representations contain error bars for the standard deviation values. Significance was designated at $95 \%$ confidence $(\alpha=0.05)$. Statistical outliers were identified as having a studentized residual $>3.5$ and removed if they met this criterion. A single data point met this criterion, in Figure 2B.

1. Patino, G. A. \& Isom, L. L. Electrophysiology and beyond: multiple roles of $\mathrm{Na}+$ channel beta subunits in development and disease. Neurosci Lett 486, 53-59 (2010). 
2. Martin, C. A., Matthews, G. D. \& Huang, C. L. Sudden cardiac death and inherited channelopathy: the basic electrophysiology of the myocyte and myocardium in ion channel disease. Heart 98, 536-543 (2012).

3. Jehle, J., Schweizer, P. A., Katus, H. A. \& Thomas, D. Novel roles for hERG K(+) channels in cell proliferation and apoptosis. Cell Death Dis 2, e193 (2011).

4. Becchetti, A. Ion channels and transporters in cancer. 1. Ion channels and cell proliferation in cancer. Am J Physiol Cell Physiol 301, C255-265 (2011).

5. Valverde, P., Kawai, T. \& Taubman, M. A. Selective blockade of voltage-gated potassium channels reduces inflammatory bone resorption in experimental periodontal disease. J Bone Miner Res 19, 155-164 (2004).

6. Davidson, S. M. \& Duchen, M. R. Imaging mitochondrial calcium signalling with fluorescent probes and single or two photon confocal microscopy. Methods $\mathrm{Mol}$ Biol 810, 219-234 (2012).

7. Shortreed, M., Bakker, E. \& Kopelman, R. Miniature sodium-selective ionexchange optode with fluorescent $\mathrm{pH}$ chromoionophores and tunable dynamic range. Anal Chem 68, 2656-2662 (1996).

8. Seiler, K. et al. Characterization of sodium-selective optode membranes based on neutral ionophores and assay of sodium in plasma. Clin Chem 37, 1350-1355 (1991)

9. Bakker, E., Buhlmann, P. \& Pretsch, E. Carrier-Based Ion-Selective Electrodes and Bulk Optodes. 1. General Characteristics. Chem Rev 97, 3083-3132 (1997).

10. Bakker, E. \& Simon, W. Selectivity of ion-sensitive bulk optodes. Analytical Chemistry 64, 1805-1812 (1992).

11. Buhlmann, P., Pretsch, E. \& Bakker, E. Carrier-Based Ion-Selective Electrodes and Bulk Optodes. 2. Ionophores for Potentiometric and Optical Sensors. Chem Rev 98, 1593-1688 (1998).

12. Dubach, J. M., Balaconis, M. K. \& Clark, H. A. Fluorescent nanoparticles for the measurement of ion concentration in biological systems. J Vis Exp (2011).

13. Harjes, D. I., Dubach, J. M., Rosenzweig, A., Das, S. \& Clark, H. A. Ion-selective optodes measure extracellular potassium flux in excitable cells. Macromol Rapid Commun 31, 217-221 (2010).

14. Dubach, J. M., Das, S., Rosenzweig, A. \& Clark, H. A. Visualizing sodium dynamics in isolated cardiomyocytes using fluorescent nanosensors. Proc Natl Acad Sci U S A 106, 16145-16150 (2009).

15. Dubach, J. M., Harjes, D. I. \& Clark, H. A. Ion-selective nano-optodes incorporating quantum dots. J Am Chem Soc 129, 8418-8419 (2007).

16. Dubach, J. M., Harjes, D. I. \& Clark, H. A. Fluorescent ion-selective nanosensors for intracellular analysis with improved lifetime and size. Nano Lett 7, 1827-1831 (2007).

17. Izeddin, I. et al. Super-resolution dynamic imaging of dendritic spines using a lowaffinity photoconvertible actin probe. PLoS One 6, e15611 (2011).

18. Luo, J., Boosalis, B. J., Thoreson, W. B. \& Margalit, E. A comparison of optical and electrophysiological methods for recording retinal ganglion cells during electrical stimulation. Curr Eye Res 37, 218-227 (2012).

19. Lamy, C. M., Sallin, O., Loussert, C. \& Chatton, J. Y. Sodium sensing in neurons with a dendrimer-based nanoprobe. ACS Nano 6, 1176-1187 (2012).

20. Lahn, M., Dosche, C. \& Hille, C. Two-photon microscopy and fluorescence lifetime imaging reveal stimulus-induced intracellular $\mathrm{Na}+$ and $\mathrm{Cl}$ - changes in cockroach salivary acinar cells. Am J Physiol Cell Physiol 300, C1323-1336 (2011).

21. Rose, C. R. High-resolution $\mathrm{Na}+$ imaging in dendrites and spines. Pflugers Arch 446, 317-321 (2003)

22. Rose, C. R. Two-photon sodium imaging in dendritic spines. Cold Spring Harb Protoc 2012, 1161-1165 (2012)

23. Ross, W. N. Understanding calcium waves and sparks in central neurons. Nat Rev Neurosci 13, 157-168 (2012).

24. Beyder, A. et al. Mechanosensitivity of Nav1.5, a voltage-sensitive sodium channel. J Physiol 588, 4969-4985 (2010).

25. Brette, F. \& Orchard, C. H. Density and sub-cellular distribution of cardiac and neuronal sodium channel isoforms in rat ventricular myocytes. Biochem Biophys Res Commun 348, 1163-1166 (2006).

26. Li, X. F. et al. Importance of $\mathrm{K}+$-dependent $\mathrm{Na}+/ \mathrm{Ca} 2+$-exchanger 2, NCKX2, in motor learning and memory. J Biol Chem 281, 6273-6282 (2006).

27. Verkerk, A. O. et al. Functional Nav1.8 channels in intracardiac neurons: the link between SCN10A and cardiac electrophysiology. Circ Res 111, 333-343 (2012).

28. Davis, R. P. et al. Cardiomyocytes derived from pluripotent stem cells recapitulate electrophysiological characteristics of an overlap syndrome of cardiac sodium channel disease. Circulation 125, 3079-3091 (2012).

29. Clay, J. R. Excitability of the squid giant axon revisited. J Neurophysiol 80, 903-913 (1998).

30. Fleidervish, I. A., Lasser-Ross, N., Gutnick, M. J. \& Ross, W. N. Na+ imaging reveals little difference in action potential-evoked $\mathrm{Na}+$ influx between axon and soma. Nat Neurosci 13, 852-860 (2010).
31. Meier, S. D., Kovalchuk, Y. \& Rose, C. R. Properties of the new fluorescent $\mathrm{Na}+$ indicator CoroNa Green: comparison with SBFI and confocal $\mathrm{Na}+$ imaging. J Neurosci Methods 155, 251-259 (2006).

32. Baartscheer, A., Schumacher, C. A. \& Fiolet, J. W. Small changes of cytosolic sodium in rat ventricular myocytes measured with SBFI in emission ratio mode. J Mol Cell Cardiol 29, 3375-3383 (1997).

33. Minta, A. \& Tsien, R. Y. Fluorescent indicators for cytosolic sodium. J Biol Chem 264, 19449-19457 (1989).

34. Ediger, M. D., Domingue, R. P. \& Fayer, M. D. Picosecond Studies of Excitation Transport in a Finite Volume - the Clustered Transport-System Octadecyl Rhodamine-B in Triton X-100 Micelles. J Chem Phys 80, 1246-1253 (1984).

35. Wolfbeis, O. S. Materials for fluorescence-based optical chemical sensors. J Mat Chem 15, 2657-2669 (2005).

36. Szmacinski, H. \& R. Lakowicz, J. Potassium and sodium measurements at clinical concentrations using phase-modulation fluorometry. Sensor Actuat B-Chem 60, 8-18 (1999).

37. Wyglądacz, K., Durnaś, M., Parzuchowski, P., Brzózka, Z. \& Malinowska, E. Miniaturized sodium-selective sensors based on silicon back-side contact structure with novel self-plasticizing ion-selective membranes. Sensor Actuat BChem 95, 366-372 (2003)

38. Tsujimura, Y., Yokoyama, M. \& Kimura, K. Practical Applicability of Silicone Rubber Membrane Sodium-Selective Electrode Based on Oligosiloxane-Modified Calix[4] arene Neutral Carrier. Anal Chem 67, 2401-2404 (1995).

39. Xie, L., Qin, Y. \& Chen, H. Y. Polymeric optodes based on upconverting nanorods for fluorescent measurements of $\mathrm{pH}$ and metal ions in blood samples. Anal Chem 84, 1969-1974 (2012)

40. Bychkova, V. \& Shvarev, A. Surface area effects on the response mechanism of ion optodes: a preliminary study. Anal Chem 81, 7416-7419 (2009).

41. Muller, M. \& Somjen, G. G. $\mathrm{Na}(+)$ and $\mathrm{K}(+)$ concentrations, extra- and intracellular voltages, and the effect of TTX in hypoxic rat hippocampal slices. J Neurophysiol 83, 735-745 (2000).

42. Shaw, R. M. \& Rudy, Y. Electrophysiologic effects of acute myocardial ischemia: a theoretical study of altered cell excitability and action potential duration. Cardiovasc Res 35, 256-272 (1997).

43. Nielsen, J. J. et al. Effects of high-intensity intermittent training on potassium kinetics and performance in human skeletal muscle. J Physiol 554, 857-870 (2004).

44. Xie, X. J., Li, X., Ge, Y. F., Qin, Y. \& Chen, H. Y. Rhodamine-based ratiometric fluorescent ion-selective bulk optodes. Sensor Actuat B-Chem 151, 71-76 (2010)

45. Seiler, K. \& Simon, W. Theoretical Aspects of Bulk Optode Membranes. Anal Chim Acta 266, 73-87 (1992).

46. Balaconis, M. K. \& Clark, H. A. Biodegradable optode-based nanosensors for in vivo monitoring. Anal Chem 84, 5787-5793 (2012).

47. Schindelin, J. et al. Fiji: an open-source platform for biological-image analysis. Nat Methods 9, 676-682 (2012).

\section{Acknowledgments}

This work was supported by the NIH through grant numbers R01 GM084366 and R01 NS081641. T.T.R. is also supported by the NIH through grant number F32 EB015847.

\section{Author contributions}

T.T.R. and H.A.C. designed the experiments. T.T.R., A.A.M. and J.M.D. performed the research. T.T.R. and H.A.C. analyzed the data and wrote the manuscript.

\section{Additional information}

Supplementary information accompanies this paper at http://www.nature.com/ scientificreports

Supporting information contains additional figures on PFN size stability, calibration data for individual fluorophores, and videos of calibration performed in dialysis tubing. Competing financial interests: The authors declare no competing financial interests. How to cite this article: Ruckh, T.T., Mehta, A.A., Dubach, J.M. \& Clark, H.A. Polymer-Free Optode Nanosensors for Dynamic, Reversible, and Ratiometric Sodium Imaging in the Physiological Range. Sci. Rep. 3, 3366; DOI:10.1038/srep03366 (2013).

his work is licensed under a Creative Commons AttributionNonCommercial-NoDerivs 3.0 Unported license. To view a copy of this license, visit http://creativecommons.org/licenses/by-nc-nd/3.0 